\title{
MULTIPLICITIES OF DIHEDRAL DISCRIMINANTS
}

\author{
DANIEL C. MAYER
}

Dedicated to the memory of Helmut Hasse

\begin{abstract}
Given the discriminant $d_{k}$ of a quadratic field $k$, the number of cyclic relative extensions $N \mid k$ of fixed odd prime degree $p$ with dihedral absolute Galois group of order $2 p$, which share a common conductor $f$, is called the multiplicity of the dihedral discriminant $d_{N}=f^{2(p-1)} d_{k}^{p}$. In this paper, general formulas for multiplicities of dihedral discriminants are derived by analyzing the $p$-rank of the ring class group $\bmod f$ of $k$. For the special case $p=3, d_{k}=-3$, an elementary proof is given additionally. The theory is illustrated by a discussion of all known discriminants of multiplicity $\geq 5$ of totally real and complex cubic fields.
\end{abstract}

\section{INTRODUCTION}

Let $p$ be an odd prime and $K \mid \mathbb{Q}$ a cyclic extension of degree $p$. Then it is well known $[9,15,7]$ that the conductor of $K$ must have the form $f=$ $p^{e} \cdot q_{1} \cdots q_{t}$, where $e=0$ or $e=2, t \geq 0$, and the $q_{i}$ are pairwise distinct rational primes satisfying $q_{i} \equiv 1(\bmod p)$ for $i=1, \ldots, t$. The discriminant of $K$ is just a power of the conductor, $d_{K}=f^{p-1}$. If, for any positive integer $f$, the number of cyclic extensions $K \mid \mathbb{Q}$ of degree $p$ which share the same conductor $f$ is denoted by $m(f)$, then

$$
\sum_{f^{\prime} \mid f} m\left(f^{\prime}\right)=\frac{1}{p-1}\left(p^{\rho}-1\right),
$$

where $\rho=\operatorname{dim}_{\mathbb{F}_{p}}\left(\mathbb{Q}^{\times}(f) / \mathbb{Q}_{f}^{\times} \cdot \mathbb{Q}^{\times}(f)^{p}\right)=\operatorname{dim}_{\mathbb{F}_{p}}\left(\operatorname{Syl}_{p} U(\mathbb{Z} / f \mathbb{Z}) \otimes_{\mathbb{Z}_{p}} \mathbb{F}_{p}\right)=t+w$ with $\mathbb{Q}^{\times}(f)=\left\{r \in \mathbb{Q}^{\times} \mid(r, f)=1\right\}, \mathbb{Q}_{f}^{\times}=\left\{r \in \mathbb{Q}^{\times} \mid r \equiv 1\left(\bmod ^{\times} f\right)\right\}$, and $w=\frac{1}{2} e$. Moebius inversion yields an explicit formula for $m(f)$ :

$$
m\left(p^{e} \cdot q_{1} \cdots q_{t}\right)=(p-1)^{t+w-1} .
$$

It is the aim of the present paper to establish similar formulas for multiplicities of discriminants in the case of non-Galois extensions $L \mid \mathbb{Q}$ of degree $p$ with dihedral normal closure $N$ of degree $2 p$. For the sake of illustration, the formulas are applied to discriminants with multiplicities up to 16 of non-Galois

Received December 10, 1990; revised April 5, 1991.

1991 Mathematics Subject Classification. Primary 11R20, 11R11, 11 R16.

Key words and phrases. Dihedral fields, quadratic ring class groups, cubic fields.

Research supported by the Austrian Science Foundation, Project Nr. J0497-PHY. 
cubic fields which occur in the most extensive recent numerical tables [10, 5 , 11].

\section{CUbiC Discriminants}

Assume that $k$ is a fixed quadratic field with discriminant $d_{k}$. If $N \mid k$ is a cyclic cubic relative extension with conductor $f$ and with absolute Galois $\operatorname{group} \operatorname{Gal}(N \mid \mathbb{Q}) \simeq S_{3}$, the symmetric group on three symbols, then [8, p. 578] $f$ must have the form

$$
f=3^{e} \cdot q_{1} \cdots q_{t}
$$

with $0 \leq e \leq 2, t \geq 0$, and pairwise distinct rational primes $q_{i} \neq 3$ satisfying $q_{i} \equiv\left(\frac{d_{k}}{q_{i}}\right)(\bmod 3)$ for $i=1, \ldots, t$. Furthermore, the 3-exponent $e$ is restricted to the values 0,2 if $d_{k} \equiv \pm 1(\bmod 3)$, and to the values 0,1 if $d_{k} \equiv 3(\bmod 9)$, but no restrictions arise for $d_{k} \equiv-3(\bmod 9)$. An integer $f$ of this form will be called an admissible conductor for the quadratic discriminant $d_{k}$.

For any positive integer $f$, define the multiplicity $m\left(d_{k}, f\right)$ of $f$ with respect to $d_{k}$ to be the number of nonconjugate cubic fields $L \mid \mathbb{Q}$ with coinciding discriminant $d_{L}=f^{2} \cdot d_{k}$. Then the following general formula for the recursive determination of multiplicities of cubic discriminants holds. This result will be proved in a more general context in $\S 3$.

Theorem 1.1. Let $f=3^{e} \cdot q_{1} \cdots q_{t}$ be an admissible conductor for the quadratic field $k$ with discriminant $d_{k}$ and 3-class rank $\rho$. Then

$$
\sum_{f^{\prime} \mid f} m\left(d_{k}, f^{\prime}\right)=\frac{1}{2}\left(3^{\rho+t+w-\delta}-1\right),
$$

where the sum runs over all divisors $f^{\prime}$ of $f$. Here,

$$
w=\left\{\begin{array}{l}
0 \text { if } e=0 \\
2 \text { if } e=2 \text { and } d_{k} \equiv-3(\bmod 9) \\
1 \text { otherwise }
\end{array}\right.
$$

and $\delta=\operatorname{dim}_{\mathbb{F}_{3}}\left(I_{k, 3}(f) / I_{k, 3}(f) \cap\left(\mathbb{Q}^{\times}(f) k_{f}^{\times} k^{\times}(f)^{3}\right)\right)$, where $k^{\times}(f)$ (resp. $\mathbb{Q}^{\times}(f)$ ) denotes the numbers in $k^{\times}$(resp. $\mathbb{Q}^{\times}$) which are coprime to $f, k_{f}^{\times}=$ $\left\{\gamma \in k^{\times} \mid \gamma \equiv 1\left(\bmod ^{\times} f\right)\right\}$ is the group of generators of the ray $\bmod f$ of $k$, and $I_{k, 3}(f)=I_{k, 3} \cap k^{\times}(f)$ with the group $I_{k, 3}$ of generators $\alpha \in k^{\times}$of all principal ideals $\alpha \mathscr{O}_{k}$ which are cubes of ideals of $k$.

\section{Pure CUbic Discriminants}

First, the complete solution of the multiplicity problem for the special case of pure cubic discriminants is obtained in a totally elementary way with the aid of the following well-known relationship [4] between the normalized radicand $D=m \cdot n^{2}$ of a pure cubic field $L=\mathbb{Q}(\sqrt[3]{D})$, where $m>n>0$ are squarefree coprime integers, and the conductor $f$ of the corresponding relative extension $N \mid k$,

$$
f= \begin{cases}3 m n & \text { if } D \not \equiv \pm 1(\bmod 9)(\text { field of Dedekind's } 1 \text { st kind }) \\ m n & \text { if } D \equiv \pm 1(\bmod 9)(\text { field of Dedekind's } 2 \text { nd kind })\end{cases}
$$


Theorem 2.1. Let $f=3^{e} \cdot q_{1} \cdots q_{t}>1$ be an admissible conductor for the special quadratic discriminant $d_{k}=-3$, i.e., $0 \leq e \leq 2, t \geq 0$, and $q_{i} \neq 3$ are arbitrary rational primes for $i=1, \ldots, t$. Put

$$
\begin{aligned}
& u=\#\left\{1 \leq i \leq t \mid q_{i} \equiv \pm 1(\bmod 9)\right\}, \\
& v=\#\left\{1 \leq i \leq t \mid q_{i} \equiv \pm 2, \pm 4(\bmod 9)\right\} .
\end{aligned}
$$

Then the multiplicity $m(f):=m(-3, f)$ of the pure cubic discriminant $-3 \cdot f^{2}$ is given by

$$
m\left(3^{e} \cdot q_{1} \cdots q_{t}\right)= \begin{cases}2^{t} & \text { if } e=2, \\ 2^{u} \cdot X_{v} & \text { if } e=1, \\ 2^{u} \cdot X_{v-1} & \text { if } e=0,\end{cases}
$$

where $X_{j}=\frac{1}{3}\left(2^{j}+(-1)^{j+1}\right)$ for all $j \geq-1$.

Moreover, the multiplicities of conductors with 3-exponents $e=0,1$ satisfy the relation

$$
m\left(q_{1} \cdots q_{t}\right)+m\left(3 \cdot q_{1} \cdots q_{t}\right)=2^{t-1} .
$$

Proof. To see that all claimed conductors are really admissible for $d_{k}=-3$, observe that $d_{k} \equiv-3(\bmod 9)$ and that the condition $q \equiv\left(\frac{d_{k}}{q}\right)(\bmod 3)$ is satisfied by every prime $q$.

First, the case $e=2$ is treated separately. The relation $f=3^{2} \cdot q_{1} \cdots q_{t}$ is equivalent to $f=3 m n, 3 \mid m n$, and thus also to $D \equiv 0(\bmod 3)$. In this case, there are $2^{t+1}$ choices for the exponent systems $1 \leq w_{0}, w_{1}, \ldots, w_{t} \leq 2$ in cubefree radicands $D=3^{w_{0}} \cdot q_{1}^{w_{1}} \cdots q_{t}^{w_{t}}$ which all share the same value of $m n=3 \cdot q_{1} \cdots q_{t}$. But only one of the two systems $\left(w_{0}, \ldots, w_{t}\right)$ and $(3-$ $w_{0}, \ldots, 3-w_{t}$ ) belongs to a normalized radicand. Hence,

$$
m\left(3^{2} \cdot q_{1} \cdots q_{t}\right)=\frac{1}{2} 2^{t+1}=2^{t} .
$$

Second, the cases $e=1$ and $e=0$ are investigated simultaneously. The relation $f=3 \cdot q_{1} \cdots q_{t}$ is equivalent to $f=3 m n, 3 \nmid m n$, and further to $D \equiv \pm 2, \pm 4(\bmod 9)$, whereas $f=q_{1} \cdots q_{t}$ is equivalent to $f=m n, 3 \nmid$ $m n$, and also to $D \equiv \pm 1(\bmod 9)$. In both cases, there are $2^{t}$ choices for exponents $1 \leq w_{1}, \ldots, w_{t} \leq 2$ in cubefree radicands $D=q_{1}^{w_{1}} \cdots q_{t}^{w_{t}}$ which all share the same value of $m n=q_{1} \cdots q_{t}$, but some of them $(D \equiv \pm 1(\bmod 9))$ give rise to conductor $f=m n$ and the others $(D \equiv \pm 2, \pm 4(\bmod 9))$ to conductor $f=3 m n$. Again, only one of the two systems $\left(w_{1}, \ldots, w_{t}\right)$ and $\left(3-w_{1}, \ldots, 3-w_{t}\right)$ belongs to a normalized radicand. (Both, the normalized and the nonnormalized radicand, are of the same Dedekind kind.) Therefore,

$$
m\left(q_{1} \cdots q_{t}\right)+m\left(3 \cdot q_{1} \cdots q_{t}\right)=\frac{1}{2} 2^{t}=2^{t-1} .
$$

To separate these two multiplicities, it is convenient to fix a value $u \geq 0$ of the number of prime divisors $q \equiv \pm 1(\bmod 9)$ of $D$ and to argue by induction with respect to the number $v \geq 0$ of prime divisors $q \equiv \pm 2, \pm 4(\bmod 9)$ of $D$. Obviously, $u+v=t$.

Induction start, $v=0,1$ :

In the case $v=0$, we have $m n=q_{1} \cdots q_{u}$ with $u \geq 1$ and $D \equiv \pm 1$ $(\bmod 9)$, whence

$$
\begin{aligned}
Y_{-1} & :=m\left(q_{1} \cdots q_{u}\right)=2^{u-1}, \\
Y_{0} & :=m\left(3 \cdot q_{1} \cdots q_{u}\right)=0 .
\end{aligned}
$$


In the case $v=1$, we have $m n=q_{1} \cdots q_{u} \cdot q_{u+1}$ and $D \equiv \pm 2, \pm 4(\bmod 9)$, whence

$$
\begin{gathered}
m\left(q_{1} \cdots q_{u} \cdot q_{u+1}\right)=0=Y_{0}, \\
Y_{1}:=m\left(3 \cdot q_{1} \cdots q_{u} \cdot q_{u+1}\right)=2^{u} .
\end{gathered}
$$

Induction step, $v \rightarrow v+1$ :

If the new prime factor $q_{u+v+1} \equiv \pm 2, \pm 4(\bmod 9)$ and its square are multiplied by a radicand $D \equiv \pm 1(\bmod 9)$, then there are generated two new radicands $D \cdot q_{u+v+1}^{w_{u+v+1}} \equiv \pm 2, \pm 4(\bmod 9)$ with $1 \leq w_{u+v+1} \leq 2$. However, if they are multiplied by a radicand $D \equiv \pm 2, \pm 4(\bmod 9)$, then one of the two new radicands is congruent $\pm 1(\bmod 9)$ (the one, where $q_{u+v+1}^{w_{u+v+1}}$ represents the square of $D$ in the group $U(\mathbb{Z} / 9 \mathbb{Z}) /\{ \pm 1\} \simeq C(3))$ and the other is congruent $\pm 2, \pm 4(\bmod 9)$. Thus,

$$
\begin{aligned}
m\left(q_{1} \cdots q_{u+v+1}\right) & =m\left(3 \cdot q_{1} \cdots q_{u+v}\right)=: Y_{v}, \\
Y_{v+1}:=m\left(3 \cdot q_{1} \cdots q_{u+v+1}\right) & =m\left(3 \cdot q_{1} \cdots q_{u+v}\right)+2 \cdot m\left(q_{1} \cdots q_{u+v}\right) \\
& =Y_{v}+2 Y_{v-1} .
\end{aligned}
$$

Consequently, the numbers $Y_{j}(j \geq-1)$ satisfy a binary linear recursion, $Y_{j+1}=Y_{j}+2 Y_{j-1}$ for $j \geq 0$, with initial values $Y_{-1}=2^{u-1}$ and $Y_{0}=0$. This recursion can be solved by diagonalization of the corresponding matrix $M$ in the equation

$$
\left(\begin{array}{c}
Y_{j+1} \\
Y_{j}
\end{array}\right)=\left(\begin{array}{ll}
1 & 2 \\
1 & 0
\end{array}\right) \cdot\left(\begin{array}{c}
Y_{j} \\
Y_{j-1}
\end{array}\right)
$$

The characteristic polynomial of $M$ is (denoting by $I$ the identity matrix)

$$
\operatorname{det}(x \cdot I-M)=x^{2}-x-2=(x+1) \cdot(x-2) .
$$

The eigenspaces of $M$ with respect to the eigenvalues -1 and 2 are

$$
\operatorname{ker}(M+I)=\mathbb{Q} \cdot\left(\begin{array}{c}
1 \\
-1
\end{array}\right), \quad \operatorname{ker}(M-2 \cdot I)=\mathbb{Q} \cdot\left(\begin{array}{l}
2 \\
1
\end{array}\right)
$$

and $M$ becomes diagonal under the transformation

$$
T^{-1} \cdot M \cdot T=\frac{1}{3}\left(\begin{array}{cc}
1 & -2 \\
1 & 1
\end{array}\right) \cdot\left(\begin{array}{ll}
1 & 2 \\
1 & 0
\end{array}\right) \cdot\left(\begin{array}{cc}
1 & 2 \\
-1 & 1
\end{array}\right)=\left(\begin{array}{cc}
-1 & 0 \\
0 & 2
\end{array}\right):=\Delta .
$$

Therefore, the solution of the recursion can be represented in the form

$$
\begin{aligned}
\left(\begin{array}{c}
Y_{j} \\
Y_{j-1}
\end{array}\right) & =M^{j} \cdot\left(\begin{array}{c}
Y_{0} \\
Y_{-1}
\end{array}\right)=T \cdot \Delta^{j} \cdot T^{-1} \cdot\left(\begin{array}{c}
Y_{0} \\
Y_{-1}
\end{array}\right) \\
& =\frac{1}{3} 2^{u}\left(\begin{array}{c}
(-1)^{j+1}+2^{j} \\
(-1)^{j}+2^{j-1}
\end{array}\right) \text { for } j \geq 0 .
\end{aligned}
$$

Hence, the solution is $Y_{j}=2^{u} \cdot X_{j}$ with $X_{j}:=\frac{1}{3}\left(2^{j}+(-1)^{j+1}\right)$ for all $j \geq$ -1 .

Remark. The proof of Theorem 2.1 used a very special elementary technique which cannot be applied to other types of cubic fields. In the next section, this result will be rederived as a particular instance of a much more general formula, which is deduced by completely different methods, using ring class groups of quadratic number fields. 
Examples. As an application, the minimal occurrences of some higher multiplicities of pure cubic discriminants are determined. The first three cases appear in tables of complex cubic fields $[1,5,12]$ which are ordered by discriminants. The further ones are constructed by means of Theorem 2.1, taking the smallest possible prime factors. These examples show that the normalized radicands associated with a fixed discriminant of higher multiplicity are spread rather widely in a table of pure cubic fields which is ordered by radicands, such as [11].

1. $m(f)=2$ for $f=3^{2} \cdot 2, d_{L}=-972$, occurs in [1], with associated radicands $D=6,12$, and $e=2, u=0, v=1$.

2. $m(f)=4$ for $f=3^{2} \cdot 2 \cdot 5, d_{L}=-24300$, occurs in [12], with associated radicands $D=30,60,90,150$, and $e=2, u=0, v=2$.

3. $m(f)=3$ for $f=3 \cdot 2 \cdot 5 \cdot 7, d_{L}=-132300$, occurs in [5], with associated radicands $D=70,140,490$, and $e=1, u=0, v=3$.

4. $m(f)=8$ for $f=3^{2} \cdot 2 \cdot 5 \cdot 7, d_{L}=-1190700$, occurs in [11], with associated radicands $D=210,420,630,1050,1260,1470,2100$, 2940 , and $e=2, u=0, v=3$.

5. $m(f)=5$ for $f=3 \cdot 2 \cdot 5 \cdot 7 \cdot 11, d_{L}=-16008300$, occurs in [11], with associated radicands $D=770,3850,7700,10780,16940$, and $e=1, u=0, v=4$.

6. $m(f)=6$ for $f=3 \cdot 2 \cdot 5 \cdot 7 \cdot 17, d_{L}=-38234700$, occurs in [11], with associated radicands $D=1190,2380,8330,11900,20230$, 40460 , and $e=1, u=1, v=3$.

7. However, $m(f)=7$ will never occur, since 7 is not a member of the sequence $\left(X_{j}\right)_{j \geq-1}$ in Theorem 2.1. The same is true for $m(f)=$ $9,13,14,15$.

8. $m(f)=16$ for $f=3^{2} \cdot 2 \cdot 5 \cdot 7 \cdot 11, d_{L}=-144074700$, occurs in [11], with associated radicands $D=2310,4620,6930,11550,13860$, $16170,23100,25410,32340,34650,48510,50820,69300,76230$, 80850,97020 , and $e=2, u=0, v=4$.

9. $m(f)=11$ for $f=3 \cdot 2 \cdot 5 \cdot 7 \cdot 11 \cdot 13, d_{L}=-2705402700$, with associated radicands $D=10010,20020,70070,100100,110110$, 260260, 350350, 550550, 650650, 700700, 910910, and $e=1, u=$ $0, v=5$.

10. $m(f)=10$ for $f=3 \cdot 2 \cdot 5 \cdot 7 \cdot 11 \cdot 17, d_{L}=-4626398700$, with associated radicands $D=13090,65450,130900,183260,222530$, $287980,458150,719950,1007930,1112650$, and $e=1, u=1$, $v=4$.

11. $m(f)=12$ for $f=3 \cdot 2 \cdot 5 \cdot 7 \cdot 17 \cdot 19, d_{L}=-13802726700$, with associated radicands $D=22610,45220,158270,226100,384370$, $429590,768740,791350,859180,1582700,2690590,3007130$, and $e=1, u=2, v=3$.

\section{DiHEDRAL DISCRIMINANTS}

As before, assume that $k$ is a fixed quadratic field with discriminant $d_{k}$. With $p$ an odd rational prime, let $N \mid k$ be a cyclic relative extension of degree $p$ with conductor $f$ and absolute Galois $\operatorname{group} \operatorname{Gal}(N \mid \mathbb{Q}) \simeq D_{p}$, the dihedral group of order $2 p$. Then $f$ is a rational integer and [2] must have the form

$$
f=p^{e} \cdot q_{1} \cdots q_{t}
$$


with $0 \leq e \leq 2, t \geq 0$, and pairwise distinct rational primes $q_{i} \neq p$ satisfying $\bar{q}_{i} \equiv\left(\frac{d_{k}}{q_{i}}\right)(\bmod p)$ for $i=1, \ldots, t$. Furthermore, the $p$-exponent $e$ is restricted to the values 0,2 if $p$ is unramified in $k$, and to the values 0,1 if $p \mid d_{k}$, except for the special configuration where $p=3$ and $d_{k} \equiv-3$ $(\bmod 9)$. An integer $f$ of this form will be called a $p$-admissible conductor for the quadratic discriminant $d_{k}$.

For any positive integer $f$, define the $p$-multiplicity $m_{p}\left(d_{k}, f\right)$ of $f$ with respect to $d_{k}$ to be the number of nonisomorphic fields $L \mid \mathbb{Q}$ of degree $p$ sharing the same discriminant $d_{L}=f^{p-1} \cdot d_{k}^{(p-1) / 2}$. By the translation theorem of Galois theory, $m_{p}\left(d_{k}, f\right)$ is also the number of cyclic extensions $N \mid k$ of degree $p$ with absolute group $\operatorname{Gal}(N \mid \mathbb{Q}) \simeq D_{p}$ sharing the common conductor $f$.

Remark. If $f$ is not a $p$-admissible conductor for $d_{k}$, then certainly $m_{p}\left(d_{k}, f\right)$ $=0$. But on the other hand, $m_{p}\left(d_{k}, f\right)$ may be zero even for a $p$-admissible conductor $f$ for $d_{k}$, as will be shown for infinite families of fields in Corollaries 3.2 and 3.3.

Denote by $\mathscr{J}_{k}(f)$ the group of (fractional) ideals of $k$ coprime to $f$, and by $\mathscr{R}_{k, f}=\left\{\alpha \mathscr{O}_{k} \mid \alpha \in \mathbb{Q}^{\times}(f) \cdot k_{f}^{\times}\right\}$the so-called ring $\bmod f$ of $k$, where $\mathbb{Q}^{\times}(f)$ denotes the numbers in $\mathbb{Q}^{\times}$which are coprime to $f$, and $k_{f}^{\times}=\left\{\gamma \in k^{\times} \mid \gamma \equiv 1\right.$ $\left.\left(\bmod ^{\times} f\right)\right\}$ is the group of generators of the ray $\bmod f$ of $k$. Then, by the Artin reciprocity law of class field theory [8], $m_{p}\left(d_{k}, f\right)$ can be interpreted as the number of subgroups $\mathscr{H}$ of index $p$ of $\mathscr{I}_{k}(f)$ which contain $\mathscr{R}_{k, f}$ but not $\mathscr{R}_{k, f^{\prime}}$ for any proper divisor $f^{\prime} \mid f, f^{\prime} \neq f$. With the aid of this fact, the following formula for the recursive determination of multiplicities of dihedral discriminants can be derived.

Theorem 3.1. Let $f=p^{e} \cdot q_{1} \cdots q_{t}$ be a $p$-admissible conductor for a given quadratic field $k$ with discriminant $d_{k}$ and with $p$-class rank $\rho=\rho_{k}(p)$. Then

$$
\sum_{f^{\prime} \mid f} m_{p}\left(d_{k}, f^{\prime}\right)=\frac{1}{p-1}\left(p^{\rho+t+w-\delta}-1\right),
$$

where the sum runs over all divisors $f^{\prime}$ of $f$. Here,

$$
w= \begin{cases}0 & \text { if } e=0, \\ 2 & \text { if } e=2, p=3, d_{k} \equiv-3(\bmod 9), \\ 1 & \text { otherwise },\end{cases}
$$

and $\delta=\delta(f)=\operatorname{dim}_{\mathbb{F}_{p}}\left(I_{k, p}(f) / I_{k, p}(f) \cap\left(\mathbb{Q}^{\times}(f) k_{f}^{\times} k^{\times}(f)^{p}\right)\right)$, where the numbers in $k^{\times}$which are coprime to $f$ are denoted by $k^{\times}(f)$, and $I_{k, p}(f)=$ $I_{k, p} \cap k^{\times}(f)$ with the group $I_{k, p}$ of generators $\alpha \in k^{\times}$of all principal ideals $\alpha \mathscr{O}_{k}$ which are pth powers of ideals of $k$.

Proof. Since the ring class group $\bmod f$ of $k, \mathscr{J}_{k}(f) / \mathscr{R}_{k, f}$, is an abelian group, its $p$-elementary subgroup is isomorphic to $\mathscr{I}_{k}(f) /\left(\mathscr{R}_{k, f} \cdot \mathscr{I}_{k}(f)^{p}\right)$. Hence, any subgroup $\mathscr{H}$ of index $p$ in $\mathscr{I}_{k}(f)$ which contains $\mathscr{R}_{k, f}$ must in fact be an intermediate group $\mathscr{R}_{k, f} \cdot \mathscr{I}_{k}(f)^{p}<\mathscr{H}<\mathscr{I}_{k}(f)$. Therefore,

$$
\sum_{f^{\prime} \mid f} m_{p}\left(d_{k}, f^{\prime}\right)=\#\left\{\mathscr{H}<\mathscr{I}_{k}(f) \mid\left(\mathscr{I}_{k}(f): \mathscr{H}\right)=p, \mathscr{R}_{k, f} \cdot \mathscr{I}_{k}(f)^{p}<\mathscr{H}\right\}
$$


is exactly the number of the hyperplanes (subspaces of codimension 1) of the $p$-elementary ring class group $\bmod f$ of $k$, viewed as a vector space over the finite field $\mathbb{F}_{p}$. But this number equals $\frac{1}{p-1}\left(p^{\rho^{\prime}}-1\right)$, where $\rho^{\prime}=$ $\operatorname{dim}_{\mathbf{F}_{p}}\left(\mathscr{J}_{k}(f) / \mathscr{R}_{k, f} \cdot \mathscr{I}_{k}(f)^{p}\right)$ is the $p$-rank of $\mathscr{I}_{k}(f) / \mathscr{R}_{k, f}$.

If $\mathscr{P}_{k}(f)=\mathscr{P}_{k} \cap \mathscr{I}_{k}(f)$ denotes the principal ideals of $k$ coprime to $f$, then the factorization relation of elementary abelian $p$-groups,

$$
\begin{aligned}
\mathscr{I}_{k}(f) / \mathscr{P}_{k}(f) \cdot \mathscr{I}_{k}(f)^{p} \\
\quad \simeq\left(\mathscr{I}_{k}(f) / \mathscr{R}_{k, f} \cdot \mathscr{I}_{k}(f)^{p}\right) /\left(\mathscr{P}_{k}(f) \cdot \mathscr{I}_{k}(f)^{p} / \mathscr{R}_{k, f} \cdot \mathscr{I}_{k}(f)^{p}\right)
\end{aligned}
$$

is equivalent to a direct product relation

$$
\begin{aligned}
\mathscr{I}_{k}(f) / \mathscr{R}_{k, f} \cdot \mathscr{I}_{k}(f)^{p} \\
\quad \simeq\left(\mathscr{I}_{k}(f) / \mathscr{P}_{k}(f) \cdot \mathscr{I}_{k}(f)^{p}\right) \times\left(\mathscr{P}_{k}(f) \cdot \mathscr{I}_{k}(f)^{p} / \mathscr{R}_{k, f} \cdot \mathscr{I}_{k}(f)^{p}\right),
\end{aligned}
$$

where the first factor is isomorphic to the $p$-elementary class group of $k$. Further, the homomorphism $k^{\times} \rightarrow \mathscr{P}_{k}, \alpha \mapsto \alpha \mathscr{O}_{k}$ induces an isomorphism

$$
\mathscr{P}_{k}(f) \cdot \mathscr{I}_{k}(f)^{p} / \mathscr{R}_{k, f} \cdot \mathscr{J}_{k}(f)^{p} \simeq k^{\times}(f) /\left(\mathbb{Q}^{\times}(f) \cdot k_{f}^{\times} \cdot I_{k, p}(f)\right) .
$$

Finally, it is well known that the local description of the congruence relation $\bmod ^{\times} f$ yields an isomorphism

$$
\begin{aligned}
k^{\times}(f) / \mathbb{Q}^{\times}(f) \cdot k_{f}^{\times} & \\
\quad & \left(k^{\times}(f) / k_{f}^{\times}\right) /\left(\mathbb{Q}^{\times}(f) / \mathbb{Q}^{\times}(f) \cap k_{f}^{\times}\right) \simeq U\left(\mathscr{O}_{k} / f \mathscr{O}_{k}\right) / U(\mathbb{Z} / f \mathbb{Z})
\end{aligned}
$$

and that the $p$-elementary subgroup of $U\left(\mathscr{O}_{k} / f \mathscr{O}_{k}\right) / U(\mathbb{Z} / f \mathbb{Z})$, which is isomorphic to $k^{\times}(f) / \mathbb{Q}^{\times}(f) \cdot k_{f}^{\times} \cdot k^{\times}(f)^{p}$, has $p$-rank equal $t+w[2,6,8]$, whence $\rho^{\prime}=\rho+t+w-\delta$ with

$$
\delta=\operatorname{dim}_{\mathbb{F}_{p}}\left(I_{k, p}(f) / I_{k, p}(f) \cap\left(\mathbb{Q}^{\times}(f) \cdot k_{f}^{\times} \cdot k^{\times}(f)^{p}\right)\right) .
$$

Remarks. 1. For totally real dihedral fields, a further decomposition of the essential index $p^{\delta}$ into two parts is useful for practical purposes:

$p^{\delta}=\left(I_{k, p}(f): I_{k, p}(f) \cap\left(\mathbb{Q}^{\times}(f) k_{f}^{\times} U_{k} k^{\times}(f)^{p}\right)\right) \cdot\left(U_{k}: U_{k} \cap\left(\mathbb{Q}^{\times}(f) k_{f}^{\times} k^{\times}(f)^{p}\right)\right)$,

where the first part involves only the principal $p$ th powers of ideals which represent $\rho$ generating classes of order $p$ of $k$, and the second part concerns exclusively the fundamental unit of $k$. Here, $U_{k}$ denotes the unit group of $k$. 2. The proof of Theorem 3.1 and the previous remark show that generally $\delta(f) \leq \min (\rho, t+w)$ (in particular, $\delta(f)=0$ when $\rho=0$ ) in the cases $d_{k}<-3$ or $p \geq 5, d_{k}=-3$, and $\delta(f) \leq \min (\rho+1, t+w)$ in the cases $d_{k}>0$ or $p=3, d_{k}=-3$.

Note. It should be pointed out that the proof of Theorem 3.1 does not use the full ray class group $\bmod f$ of $k$ but only the ring class group $\bmod f$ of $k$, which is obtained from the former by factoring out the part invariant under the generating automorphism of $\operatorname{Gal}(k \mid \mathbb{Q})[6,8]$. Otherwise, there would be counted all cyclic extensions $N \mid k$ of degree $p$ sharing the common conductor $f$, those with group $\operatorname{Gal}(N \mid \mathbb{Q}) \simeq D_{p}$ as well as those with $\operatorname{group} \operatorname{Gal}(N \mid \mathbb{Q}) \simeq$ $C(2) \times C(p)$ (composita of $k$ with cyclic extensions $K \mid \mathbb{Q}$ of degree $p$ ). 
The only case where the sum in the formula of Theorem 3.1 consists of a single term is the unramified case $f=1$, which was treated in $[8$, p. 581] and [14] already.

Corollary 3.1. The number of unramified cyclic extensions $N \mid k$ of degree $p$ is

$$
m_{p}\left(d_{k}, 1\right)=\frac{1}{p-1}\left(p^{\rho}-1\right) .
$$

Proof. If $f=1$, that is, $t=0$ and $w=0$, then we have $\delta(f)=0$, since $\delta(f) \leq \min (\rho+1, t+w)=0$, or also simply since $k_{f}^{\times}=k^{\times}$.

In all other cases, single multiplicities can be obtained by Moebius inversion.

Theorem 3.2 (General multiplicity formula when $w \leq 1$ ). Assume that $f=$ $p^{e} \cdot q_{1} \cdots q_{t}>1$ with $w \leq 1$ is a p-admissible conductor for the quadratic field $k$, put $q_{t+1}=p^{e}$ when $w=1$, and define $\delta_{\max }=\max \left\{\delta\left(q_{i_{1}} \cdots q_{i_{s}}\right) \mid 0 \leq s \leq\right.$ $\left.t+w, 1 \leq i_{1}<\cdots<i_{s} \leq t+w\right\}$. Then

$$
\begin{aligned}
& m_{p}\left(d_{k}, q_{1} \cdots q_{t+w}\right) \\
&=p^{\rho} \cdot \frac{1}{p^{\delta_{\max }}} \cdot\left[\begin{array}{l}
(p-1)^{t+w-1} \\
\left.\quad+\sum_{s=0}^{t+w}(-1)^{t+w-s} \cdot p^{s} \sum_{1 \leq i_{1}<\cdots<i_{s} \leq t+w} \frac{p^{\delta_{\max }-\delta\left(q_{i_{1}} \cdots q_{i_{s}}\right)}-1}{p-1}\right] .
\end{array}\right.
\end{aligned}
$$

Proof. Observe that, for fixed $p$ and $d_{k}$, the general formula in Theorem 3.1,

$$
\sum_{f^{\prime} \mid f} m_{p}\left(d_{k}, f^{\prime}\right)=\frac{1}{p-1}\left(p^{\rho+t+w-\delta}-1\right),
$$

can be viewed as the sum relation $\sum_{f^{\prime} \mid f} m\left(f^{\prime}\right)=n(f)$ between two integervalued functions $n(f)=\frac{1}{p-1}\left(p^{\rho+t(f)+w(f)-\delta(f)}-1\right)$ and $m\left(f^{\prime}\right)=m_{p}\left(d_{k}, f^{\prime}\right)$. Hence, an application of the Moebius inversion formula yields an expression for a single multiplicity,

$$
\begin{aligned}
m\left(q_{1} \cdots q_{t+w}\right)= & \sum_{s=0}^{t+w} \sum_{1 \leq i_{1}<\cdots<i_{s} \leq t+w} \mu\left(\frac{q_{1} \cdots q_{t+w}}{q_{i_{1}} \cdots q_{i_{s}}}\right) \cdot n\left(q_{i_{1}} \cdots q_{i_{s}}\right) \\
= & \sum_{s=0}^{t+w} \sum_{1 \leq i_{1}<\cdots<i_{s} \leq t+w}(-1)^{t+w-s} \cdot \frac{1}{p-1}\left(p^{\rho} \cdot p^{s} \cdot \frac{1}{p^{\delta\left(q_{i_{1}} \cdots q_{i_{s}}\right)}}-1\right) \\
= & \sum_{s=0}^{t+w} \sum_{1 \leq i_{1}<\cdots<i_{s} \leq t+w}(-1)^{t+w-s} \cdot \frac{1}{p-1} \cdot p^{\rho} \cdot p^{s} \cdot \frac{1}{p^{\delta\left(q_{i_{1}} \cdots q_{i_{s}}\right)}} \\
& -\frac{1}{p-1} \sum_{s=0}^{t+w}(-1)^{t+w-s} \sum_{1 \leq i_{1}<\cdots<i_{s} \leq t+w} 1 .
\end{aligned}
$$


Now the second double sum equals $\sum_{s=0}^{t+w}(-1)^{t+w-s} \cdot\left(\begin{array}{c}t+w \\ s\end{array}\right)=0$; hence,

$$
\begin{aligned}
m\left(q_{1} \cdots q_{t+w}\right)= & \frac{1}{p-1} \cdot p^{\rho} \cdot \frac{1}{p^{\delta_{\max }}} \\
& \cdot\left[\sum_{s=0}^{t+w}(-1)^{t+w-s} \cdot p^{s} \sum_{1 \leq i_{1}<\cdots<i_{s} \leq t+w} 1\right. \\
& \left.\quad+\sum_{s=0}^{t+w} \sum_{1 \leq i_{1}<\cdots<i_{s} \leq t+w}(-1)^{t+w-s} \cdot p^{s} \cdot\left(p^{\delta_{\max }-\delta\left(q_{i_{1}} \cdots q_{i_{s}}\right)}-1\right)\right],
\end{aligned}
$$

where the first double sum equals $\sum_{s=0}^{t+w}(-1)^{t+w-s} \cdot p^{s} \cdot\left(\begin{array}{c}t+w \\ s\end{array}\right)=(p-1)^{t+w}$; thus,

$$
\begin{aligned}
m\left(q_{1} \cdots q_{t+w}\right)= & \frac{1}{p-1} \cdot p^{\rho} \cdot \frac{1}{p^{\delta_{\max }}} \\
& \cdot\left[(p-1)^{t+w}\right. \\
& \left.+\sum_{s=0}^{t+w} \sum_{1 \leq i_{1}<\cdots<i_{s} \leq t+w}(-1)^{t+w-s} \cdot p^{s} \cdot\left(p^{\delta_{\max }-\delta\left(q_{i_{1}} \cdots q_{i_{s}}\right)}-1\right)\right] .
\end{aligned}
$$

This formula can be simplified further, since the indices for composed conductors, $\delta\left(q_{i_{1}} \cdots q_{i_{s}}\right)$ with $0 \leq s \leq t+w$ and $1 \leq i_{1}<\cdots<i_{s} \leq t+w$, can be determined from those for prime conductors, $\delta\left(q_{i}\right)$ with $1 \leq i \leq t+w$.

Corollary 3.2 (The special cases of $\delta_{\max }=0,1$ when $w \leq 1$ ). Let $f=$ $p^{e} \cdot q_{1} \cdots q_{t}>1$ with $w \leq 1$ be a p-admissible conductor for the quadratic field $k$, put $q_{t+1}=p^{e}$ when $w=1$, suppose that $\delta_{\max }<2$, and put $u=$ $\#\left\{1 \leq i \leq t+w \mid \delta\left(q_{i}\right)=0\right\}$, i.e., $v:=t+w-u$ is the number of "bad" primes.

1 (The case without constraints from principal $p$ th ideal powers [8, p. 582]). If $u=t+w$, then $\delta_{\max }=0$ and

$$
m_{p}\left(d_{k}, q_{1} \cdots q_{t+w}\right)=p^{\rho} \cdot(p-1)^{t+w-1} .
$$

2 (Restrictions caused by principal $p$ th ideal powers). If $0 \leq u \leq t+w-1$, then $\delta_{\max }=1$ and

$$
m_{p}\left(d_{k}, q_{1} \cdots q_{t+w}\right)=p^{\rho} \cdot(p-1)^{u} \cdot \frac{(p-1)^{v-1}-(-1)^{v-1}}{p} .
$$

In particular, $m_{p}\left(d_{k}, q_{1} \cdots q_{t+w}\right)=0$ for $v=1$.

Proof. 1. If $u=t+w$, then $\delta\left(q_{i}\right)=0$ for all $1 \leq i \leq t+w$, and consequently $\delta\left(q_{i_{1}} \cdots q_{i_{s}}\right)=0$ for all $0 \leq s \leq t+w$ and $1 \leq i_{1}<\cdots<i_{s} \leq t+w$, whence $\delta_{\max }=0$. In this case, the formula of Theorem 3.2 immediately degenerates to

$$
m_{p}\left(d_{k}, q_{1} \cdots q_{t+w}\right)=p^{\rho} \cdot(p-1)^{t+w-1} .
$$

2. If $0 \leq u \leq t+w-1$, then $\delta\left(q_{i}\right)=1$ for some $1 \leq i \leq t+w$, and thus $\delta_{\max }=1$. In this case, it turns out that

$$
\#\left\{1 \leq i_{1}<\cdots<i_{s} \leq t+w \mid \delta\left(q_{i_{1}} \cdots q_{i_{s}}\right)=0\right\}=\left(\begin{array}{l}
u \\
s
\end{array}\right)
$$


for all $0 \leq s \leq t+w$, whence

$$
\begin{aligned}
m_{p}( & \left.d_{k}, q_{1} \cdots q_{t+w}\right) \\
& =p^{\rho} \cdot \frac{1}{p^{\delta_{\max }}} \cdot\left[(p-1)^{t+w-1}\right. \\
& \left.+\sum_{s=0}^{t+w}(-1)^{t+w-s} \cdot p^{s} \sum_{1 \leq i_{1}<\cdots<i_{s} \leq t+w} \frac{p^{\delta_{\max }-\delta\left(q_{i_{1}} \cdots q_{i_{s}}\right)}-1}{p-1}\right] \\
& =p^{\rho} \cdot \frac{1}{p} \cdot\left[(p-1)^{t+w-1}+\sum_{s=0}^{t+w}(-1)^{t+w-s} \cdot p^{s} \cdot\left(\begin{array}{l}
u \\
s
\end{array}\right) \cdot \frac{p-1}{p-1}\right] \\
& =p^{\rho} \cdot \frac{1}{p} \cdot\left[(p-1)^{t+w-1}+(-1)^{t+w-u} \sum_{s=0}^{u}\left(\begin{array}{l}
u \\
s
\end{array}\right) \cdot(-1)^{u-s} \cdot p^{s}\right] \\
& =p^{\rho} \cdot \frac{1}{p} \cdot\left[(p-1)^{t+w-1}+(-1)^{t+w-u}(p-1)^{u}\right] \\
& =p^{\rho} \cdot(p-1)^{u} \cdot \frac{(p-1)^{t+w-1-u}+(-1)^{t+w-u}}{p} .
\end{aligned}
$$

\begin{tabular}{|c|c|c|c|c|c|c|c|c|c|c|}
\hline$t+w$ & $u=$ & 0 & 1 & 2 & 3 & 4 & 5 & 6 & 7 & 8 \\
\hline 1 & & 0 & 1 & & & & & & & \\
\hline 2 & & 1 & 0 & 2 & & & & & & \\
\hline 3 & & 1 & 2 & 0 & 4 & & & & & \\
\hline 4 & & 3 & 2 & 4 & 0 & 8 & & & & \\
\hline 5 & & 5 & 6 & 4 & 8 & 0 & 16 & & & \\
\hline 6 & & 11 & 10 & 12 & 8 & 16 & 0 & 32 & & \\
\hline 7 & & 21 & 22 & 20 & 24 & 16 & 32 & 0 & 64 & \\
\hline 8 & & 43 & 42 & 44 & 40 & 48 & 32 & 64 & 0 & 128 \\
\hline
\end{tabular}

The following array shows the multiplicities $m_{p}\left(d_{k}, q_{1} \cdots q_{t+w}\right)$ in dependence on the total number $t+w>0$ of prime divisors of the conductor $f>1$, and on the number $0 \leq u \leq t+w$ of those "nice" primes which do not cause restrictions, for the special case $p=3$ and $\rho=0$. For positive 3-rank $\rho>0$ of $k$, the numbers must only be multiplied by $3^{\rho}$, provided that still $\delta_{\max }<2$.

Example 1. For pure cubic fields $L=\mathbb{Q}(\sqrt[3]{D})$, the formulas with $w=e \leq 1$ of Theorem 2.1 in the previous section, which were proved by elementary methods, can be reobtained here as the special case $p=3, d_{k}=-3, \rho=0$, and $u=\#\left\{1 \leq i \leq t+w \mid q_{i} \equiv \pm 1(\bmod 9)\right\}$, since for any $1 \leq i \leq t+w$ the condition $\delta\left(q_{i}\right)=0$ is equivalent to $\zeta_{3} \in \mathbb{Q}^{\times}\left(q_{i}\right) \cdot k_{q_{i}}^{\times} \cdot k^{\times}\left(q_{i}\right)^{3}$ and hence to $q_{i} \equiv \pm 1(\bmod 9)$ :

$$
m_{3}\left(-3, q_{1} \cdots q_{t+w}\right)=2^{u} \cdot \frac{2^{v+w-1}-(-1)^{v+w-1}}{3},
$$

where $v:=t-u$ and $v+w$ is the number of bad primes.

Finally, supplementary formulas must be established for the special case $p=$ $3, d_{k} \equiv-3(\bmod 9), w=2$. 
Theorem 3.3 (General multiplicity formula when $w=2$ ). Let $f=3^{2} \cdot q_{1} \cdots q_{t}$ with $w=2$ be a 3-admissible conductor for the quadratic field $k$. Then

$$
\begin{aligned}
& m_{3}\left(d_{k}, 3^{2} \cdot q_{1} \cdots q_{t}\right) \\
& \quad=3^{\rho} \sum_{s=0}^{t}(-1)^{t-s} \cdot 3^{s} \sum_{1 \leq i_{1}<\cdots<i_{s} \leq t} \frac{1}{2}\left(3^{2-\delta\left(3^{2} \cdot q_{i_{1}} \cdots q_{i_{s}}\right)}-3^{1-\delta\left(3 \cdot q_{i_{1}} \cdots q_{i_{s}}\right)}\right) .
\end{aligned}
$$

Proof. An application of the Moebius inversion formula to the sum relation $n(f)=\sum_{f^{\prime} \mid f} m\left(f^{\prime}\right)$ in Theorem 1.1, where $n(f)=\frac{1}{2}\left(3^{\rho+t(f)+w(f)-\delta(f)}-1\right)$ and $m\left(f^{\prime}\right)=m_{3}\left(d_{k}, f^{\prime}\right)$, yields an expression for the single multiplicity of the conductor $f=3^{2} \cdot q_{1} \cdots q_{t}$ :

$$
m\left(3^{2} \cdot q_{1} \cdots q_{t}\right)=\sum_{v=0}^{2} \sum_{s=0}^{t} \sum_{1 \leq i_{1}<\cdots<i_{s} \leq t} \mu\left(\frac{3^{2} \cdot q_{1} \cdots q_{t}}{3^{v} \cdot q_{i_{1}} \cdots q_{i_{s}}}\right) \cdot n\left(3^{v} \cdot q_{i_{1}} \cdots q_{i_{s}}\right),
$$

where the Moebius function takes the values

$$
\begin{cases}0 & \text { if } v=0 \\ (-1)^{t-s+1} & \text { if } v=1 \\ (-1)^{t-s} & \text { if } v=2\end{cases}
$$

Consequently,

$$
\begin{aligned}
m\left(3^{2} \cdot q_{1} \cdots q_{t}\right)=\sum_{s=0} \sum_{1 \leq i_{1}<\cdots<i_{s} \leq t} & {\left[(-1)^{t-s+1} \cdot \frac{1}{2}\left(3^{\rho} \cdot 3^{s+1} \cdot \frac{1}{3^{\delta\left(3 \cdot q_{i_{1}} \cdots q_{i_{s}}\right)}}-1\right)\right.} \\
& \left.+(-1)^{t-s} \cdot \frac{1}{2}\left(3^{\rho} \cdot 3^{s+2} \cdot \frac{1}{3^{\delta\left(3^{2} \cdot q_{i_{1} \cdots} \cdots q_{i_{s}}\right)}}-1\right)\right] \\
= & 3^{\rho} \sum_{s=0}^{t}(-1)^{t-s} \cdot 3^{s} \sum_{1 \leq i_{1}<\cdots<i_{s} \leq t} \frac{1}{2}\left(3^{2-\delta\left(3^{2} \cdot q_{i_{1}} \cdots q_{i_{s}}\right)}-3^{1-\delta\left(3 \cdot q_{i_{1}} \cdots q_{i_{s}}\right)}\right) .
\end{aligned}
$$

Corollary 3.3 (The special cases of $\delta_{\max }=0,1$ when $w=2$ ). Let $f=$ $3^{2} \cdot q_{1} \cdots q_{t}$ with $w=2$ be a 3-admissible conductor for the quadratic field $k$. Further, redefine $\delta_{\max }=\max \left\{\delta\left(3^{v} \cdot q_{i_{1}} \cdots q_{i_{s}}\right) \mid 0 \leq v \leq 2,0 \leq s \leq t, 1 \leq\right.$ $\left.i_{1}<\cdots<i_{s} \leq t\right\}$, suppose that $\delta_{\max }<2$, and put $u=\#\left\{1 \leq i \leq t \mid \delta\left(q_{i}\right)=0\right\}$. Then

$$
\begin{aligned}
m_{3}\left(d_{k},\right. & \left.3^{2} \cdot q_{1} \cdots q_{t}\right) \\
= & \begin{cases}3^{\rho+1} \cdot 2^{t} & \text { if } \delta\left(3^{2}\right)=0, u=t, \\
3^{\rho+1} \cdot 2^{u+1} \cdot \frac{1}{3}\left(2^{t-u-1}-(-1)^{t-u-1}\right) & \text { if } \delta\left(3^{2}\right)=0,0 \leq u \leq t-1, \\
3^{\rho+1} \cdot 2^{u} \cdot \frac{1}{3}\left(2^{t-u}-(-1)^{t-u}\right) & \text { if } \delta(3)=0, \delta\left(3^{2}\right)=1, \\
3^{\rho} \cdot 2^{t} & \text { if } \delta(3)=1 .\end{cases}
\end{aligned}
$$

The first case is the unconstrained one $[8, p .582]$ where $\delta_{\max }=0$, in the second case the multiplicity is zero for $u=t-1$, in the third case for $u=t$, and the fourth case is actually independent of $u$.

Proof. 1. If $\delta\left(3^{2}\right)=0, u=t$, then clearly $\delta_{\max }=0$, and the formula in Theorem 3.3 becomes

$$
m_{3}\left(d_{k}, 3^{2} \cdot q_{1} \cdots q_{t}\right)=3^{\rho} \sum_{s=0}^{t}(-1)^{t-s} \cdot 3^{s} \cdot\left(\begin{array}{l}
t \\
s
\end{array}\right) \cdot \frac{1}{2}\left(3^{2}-3\right)=3^{\rho+1} \cdot 2^{t} .
$$


2. However, if $\delta\left(3^{2}\right)=0$ but $0 \leq u \leq t-1$, then certainly $\delta_{\max }=1$, and $\delta\left(3^{2} \cdot q_{i_{1}} \cdots q_{i_{s}}\right)=\delta\left(3 \cdot q_{i_{1}} \cdots q_{i_{s}}\right)=\delta\left(q_{i_{1}} \cdots q_{i_{s}}\right)$ for all $0 \leq s \leq t$ and $1 \leq i_{1}<\cdots<i_{s} \leq t$. In this case,

$$
\begin{aligned}
m_{3}( & \left.d_{k}, 3^{2} \cdot q_{1} \cdots q_{t}\right) \\
& =3^{\rho} \sum_{s=0}^{t}(-1)^{t-s} \cdot 3^{s} \sum_{1 \leq i_{1}<\cdots<i_{s} \leq t} \frac{1}{2}\left(3^{2-\delta\left(q_{i_{1}} \cdots q_{i_{s}}\right)}-3^{1-\delta\left(q_{i_{1}} \cdots q_{i_{s}}\right)}\right) \\
& =3^{\rho} \sum_{s=0}^{t}(-1)^{t-s} \cdot 3^{s} \sum_{1 \leq i_{1}<\cdots<i_{s} \leq t} \frac{1}{2}(3-1) \cdot 3^{1-\delta\left(q_{i_{1}} \cdots q_{i_{s}}\right)} \\
& =3^{\rho}\left[\sum_{s=0}^{t}(-1)^{t-s} \cdot 3^{s} \cdot\left(\begin{array}{l}
t \\
s
\end{array}\right)+(-1)^{t-u} \sum_{s=0}^{t}(-1)^{u-s} \cdot 3^{s} \cdot\left(\begin{array}{l}
u \\
s
\end{array}\right) \cdot(3-1)\right] \\
& =3^{\rho} \cdot\left(2^{t}+(-1)^{t-u} \cdot 2^{u} \cdot 2\right) .
\end{aligned}
$$

3. In the case $\delta(3)=0, \delta\left(3^{2}\right)=1$, we have $\delta\left(3 \cdot q_{i_{1}} \cdots q_{i_{s}}\right)=\delta\left(q_{i_{1}} \cdots q_{i_{s}}\right)$ and $\delta\left(3^{2} \cdot q_{i_{1}} \cdots q_{i_{s}}\right)=1$ for all $0 \leq s \leq t$ and $1 \leq i_{1}<\cdots<i_{s} \leq t$, whence $\delta_{\max }=1$ and

$$
\begin{aligned}
m_{3}\left(d_{k}, 3^{2} \cdot q_{1} \cdots q_{t}\right) & =3^{\rho} \sum_{s=0}^{t}(-1)^{t-s} \cdot 3^{s} \sum_{1 \leq i_{1}<\cdots<i_{s} \leq t} \frac{1}{2}\left(3-3^{1-\delta\left(q_{i_{1}} \cdots q_{i_{s}}\right)}\right) \\
& =3^{\rho} \sum_{s=0}^{t}(-1)^{t-s} \cdot 3^{s} \cdot \frac{1}{2}(3-1) \cdot\left[\left(\begin{array}{l}
t \\
s
\end{array}\right)-\left(\begin{array}{l}
u \\
s
\end{array}\right)\right] \\
& =3^{\rho}\left[\sum_{s=0}^{t}(-1)^{t-s} \cdot 3^{s} \cdot\left(\begin{array}{l}
t \\
s
\end{array}\right)-(-1)^{t-u} \sum_{s=0}^{t}(-1)^{u-s} \cdot 3^{s} \cdot\left(\begin{array}{l}
u \\
s
\end{array}\right)\right] \\
& =3^{\rho} \cdot\left(2^{t}-(-1)^{t-u} \cdot 2^{u}\right) .
\end{aligned}
$$

4. If, finally, $\delta(3)=1$, then $\delta\left(3 \cdot q_{i_{1}} \cdots q_{i_{s}}\right)=1$ and $\delta\left(3^{2} \cdot q_{i_{1}} \cdots q_{i_{s}}\right)=1$ for all $0 \leq s \leq t$ and $1 \leq i_{1}<\cdots<i_{s} \leq t$, whence $\delta_{\max }=1$ and

$$
m_{3}\left(d_{k}, 3^{2} \cdot q_{1} \cdots q_{t}\right)=3^{\rho} \sum_{s=0}^{t}(-1)^{t-s} \cdot 3^{s} \cdot\left(\begin{array}{l}
t \\
s
\end{array}\right) \cdot \frac{1}{2}(3-1)=3^{\rho} \cdot 2^{t}
$$

Example 2. For pure cubic fields $L=\mathbb{Q}(\sqrt[3]{D})$, the formula with $w=e=2$ in Theorem 2.1 of the previous section can be reobtained as the special case $d_{k}=-3, \rho=0$, and $\delta(3)=1$ :

$$
m_{3}\left(-3,3^{2} \cdot q_{1} \cdots q_{t}\right)=2^{t} .
$$

The various formulas of $\S 3$ are illustrated in the Supplements section at the end of this issue by a discussion of all known discriminants of multiplicity $\geq 5$ of totally real and complex cubic fields, which occur in the most extensive recent numerical tables [10], [5].

Final Note. It should be particularly emphasized that all the calculations which are necessary for the determination of the multiplicity $m_{p}\left(d_{k}, f\right)$ of a dihedral discriminant $d_{L}=f^{p-1} d_{k}^{(p-1) / 2}$ can be carried out entirely in the underlying quadratic field $k$. 
Given a quadratic field $k$ with $p$-class rank $\rho=\rho_{k}(p)$ and discriminant $d_{k}$, and given a $p$-admissible conductor $f$ for $d_{k}$, we have to find $\rho$ principal $p$ th powers $\alpha_{1}, \ldots, \alpha_{\rho}$ of ideals prime to $f$ which represent $\rho$ generating classes of order $p$ of the $p$-elementary class group of $k$, and additionally a fundamental unit $\eta$ of $k$ if $d_{k}>0$ or $d_{k}=-3$. Then $I_{k, p}(f)=$ $\left\langle\alpha_{1}, \ldots, \alpha_{\rho}(, \eta)\right\rangle \cdot k^{\times}(f)^{p}$.

Next, we must examine successively, if $\eta \in \mathbb{Q}^{\times}(f) \cdot k_{f}^{\times} \cdot k^{\times}(f)^{p}$ and if

$$
\alpha_{i} \in\left\langle\alpha_{1}, \ldots, \alpha_{i-1}\right\rangle \cdot U_{k} \cdot \mathbb{Q}^{\times}(f) \cdot k_{f}^{\times} \cdot k^{\times}(f)^{p} \text { for } i=1, \ldots, \rho,
$$

whence we will be able to determine $\delta\left(f^{\prime}\right)$ for all divisors $f^{\prime} \mid f$ and finally the multiplicity $m_{p}\left(d_{k}, f\right)$ of $d_{L}=f^{p-1} d_{k}^{(p-1) / 2}$, by means of Theorem 3.2 or 3.3.

In this manner it is possible to construct complete tables of complex or totally real dihedral discriminants up to a given bound, $\left|d_{L}\right|<B$, and for a fixed value of the prime $p$, by the computation of the $p$-ranks of ring class groups mod $f$ of quadratic fields $k$, varying the discriminants $d_{k}$ and the $p$-admissible conductors $f$ for each discriminant $d_{k}$.

In particular, approximations can be determined for the asymptotic densities of dihedral discriminants for various primes $p \geq 5$, similar to the densities of Davenport and Heilbronn [3] for $p=3$. As D. Shanks pointed out in his review [13] of [1], the convergence of these approximations to the asymptotic limits would be rather slow, because the really high multiplicities, which contribute the essential part to the limit, unfortunately occur in very high ranges of discriminants.

A drawback of the proposed method is that it does not seem to be suitable for obtaining generating polynomials for the non-Galois subfields $L$ of dihedral fields.

Example. For any two positive integers $m$ and $B$, let $n_{p}(m, B)$ denote the number of complex dihedral discriminants $d_{L}=f^{p-1} d_{k}^{(p-1) / 2}$ of multiplicity $m_{p}\left(d_{k}, f\right)=m$, which are bounded by $\left|d_{L}\right|<B^{(p-1) / 2}$. (Observe that dihedral discriminants are always complete $((p-1) / 2)$ th powers.)

To make the ideas in the final note more concrete, we have computed the 303968 quadratic discriminants in the range $-10^{6}<d_{k}<0$ and determined the class numbers and $p$-class ranks $\rho=\rho_{k}(p)$ of the corresponding imaginary quadratic fields $k$ for $p=3,5,7$. With the aid of this information, we can calculate the exact numbers $n_{p}(m, B)$ for $m=1, p-1, p+1$, and for any given upper bound $B \leq 10^{6}$.

(a) According to Corollary 3.1, the first component of $n_{p}(1, B)$ is the number of quadratic fields $k$ with $p$-rank $\rho=1$ and discriminant

$$
\left|d_{k}\right|=\left|d_{L}\right|^{2 /(p-1)}<B,
$$

i.e., single unramified cyclic extensions (absolute class fields) $N \mid k$ of degree $p$ with conductor $f=1$.

By Corollary 3.2,1, the second component is the number of ramified cyclic extensions (ring class fields) $N$ of degree $p$ over quadratic fields $k$ with $p$-rank $\rho=0$ (and thus $\delta=0$ ) and discriminant

$$
\left|d_{k}\right|=\left|d_{L} / f^{p-1}\right|^{2 /(p-1)}=\left|d_{L}\right|^{2 /(p-1)} / f^{2}<B / f^{2}
$$


with a single prime divisor $q_{1}$ of the conductor $f$, such that $t+w=1$. To compute this number, we examine each of the relevant quadratic discriminants $d_{k}$ for admissible prime conductors $q_{1} \equiv \pm 1(\bmod p)$, also taking into consideration the possibility of $p \mid f, w=1$.

In the case of $p=3$, we must further add the number of pure cubic discriminants $\left(d_{k}=-3\right)$ of multiplicity 1 , which can be evaluated by counting primes and products of up to three primes below certain bounds, according to Theorem 2.1, taking into account the parameter values

$$
\begin{aligned}
(e, t) & =(2,0) \text { and } \\
(e, u, v) & =(1,0,1),(1,0,2),(0,1,0),(0,0,2),(0,0,3)
\end{aligned}
$$

\begin{tabular}{|c|c|c|c|c|c|c|c|c|c|}
\hline \multirow[t]{2}{*}{$\bar{p}$} & \multirow[t]{2}{*}{$(p-1) / 2$} & \multirow[t]{2}{*}{$\bar{B}$} & \multirow[t]{2}{*}{$n_{p}(1, B)$} & \multirow{2}{*}{\multicolumn{2}{|c|}{$f=1$}} & & \multicolumn{3}{|c|}{$f>1$} \\
\hline & & & & & & & $d_{k}<-3$ & & $d_{k}=-3$ \\
\hline \multirow[t]{4}{*}{3} & 1 & $10^{3}$ & 125 & $=$ & 84 & + & 35 & + & 6 \\
\hline & & $10^{4}$ & 1396 & $=$ & 1034 & + & 343 & + & 19 \\
\hline & & $10^{5}$ & 14565 & $=$ & 11286 & + & 3215 & + & 64 \\
\hline & & $10^{6}$ & 149204 & $=$ & 118455 & + & 30559 & + & 190 \\
\hline \multirow[t]{4}{*}{5} & 2 & $10^{3}$ & 58 & $=$ & 52 & + & & 6 & \\
\hline & & $10^{4}$ & 665 & $=$ & 617 & + & & 48 & \\
\hline & & $10^{5}$ & 7099 & $=$ & 6686 & + & & 413 & \\
\hline & & $10^{6}$ & 73252 & $=$ & 69365 & + & & 3887 & \\
\hline \multirow[t]{4}{*}{7} & 3 & $10^{3}$ & 31 & $=$ & 30 & + & & 1 & \\
\hline & & $10^{4}$ & 435 & $=$ & 411 & + & & 24 & \\
\hline & & $10^{5}$ & 4709 & $=$ & 4503 & + & & 206 & \\
\hline & & $10^{6}$ & 49607 & $=$ & 47595 & + & & 2012 & \\
\hline
\end{tabular}

The result $n_{3}\left(1,10^{6}\right)=149204$, in comparison to the number 148709 claimed in [5, Table 5.2, p. 321], shows that 495 complex cubic discriminants of multiplicity 1 were missing from the original version of [5].

The series of single complex dihedral discriminants starts with -23 for $p=3$, with $+2209=(-47)^{2}$ for $p=5$, and with $-357911=(-71)^{3}$ for $p=7$. The minimal discriminants of arbitrary quintic fields with signature $(1,2)$, resp. septic fields with signature $(1,3)$, are somewhat smaller and not $((p-1) / 2)$ th powers: +1609 , resp. -184607 . Unramified extensions are dominating, $79.5 \%$ for $p=3,94.7 \%$ for $p=5$, and $95.9 \%$ for $p=7$, and this effect even seems to increase with the value of the prime $p$. The minimal examples of the rare ramified extensions are $d_{k}=-11$ with $f=2$ for $p=3$, $d_{k}=-15$ with $f=5$ for $p=5$, and $d_{k}=-7$ with $f=7$ for $p=7$.

(b) By Corollary 3.2,1, $n_{p}(p-1, B)$ is the number of $(p-1)$-tuplets of ramified cyclic extensions $N$ of degree $p$ over quadratic fields $k$ of $p$-rank $\rho=0$ and discriminant $\left|d_{k}\right|<B / f^{2}$ with two prime divisors $q_{1}, q_{2}$ of the conductor $f$, such that $w \leq 1$.

But for the special case $p=3$, the number of pure cubic discriminants of multiplicity 2 must be added. According to Theorem 2.1, we determine this number by counting primes and products of at most four primes up to certain 
bounds, taking into consideration the parameter values

$$
\begin{aligned}
(e, t) & =(2,1) \text { and } \\
(e, u, v) & =(1,1,1),(1,1,2),(0,2,0),(0,1,2),(0,1,3) .
\end{aligned}
$$

It might be of interest to list explicitly the root discriminants $\left|d_{L}\right|^{2 /(p-1)}$ for the few encountered $(p-1)$-tuplets of dihedral fields with two prime divisors of the conductor $f$, if $p=5,7$ (for $p=3$, they start with the well-known 1836 [1]).

There are 20 cases for $p=5$ :

$105875,121000,180500,287375,305767,315375,315875,360375$, $363000,496375,529375,589875,605000,650375,771375,786500$, $814088,840500,841000,902500$,

and 7 cases for $p=7$ :

$57967,288463,576583,634207,695604,985439,994903$.

\begin{tabular}{cc|c|ccccc}
\hline$p$ & $(p-1) / 2$ & $B$ & $n_{p}(p-1, B)$ & & & $f>1$ & \\
& & & & & $d_{k}<-3$ & & $d_{k}=-3$ \\
\hline 3 & 1 & $10^{3}$ & 1 & $=$ & 0 & + & 1 \\
& & $10^{4}$ & 12 & $=$ & 10 & + & 2 \\
& & $10^{5}$ & 167 & $=$ & 157 & + & 10 \\
& & $10^{6}$ & 1683 & $=$ & 1639 & + & 44 \\
5 & 2 & $10^{6}$ & 20 & $=$ & & 20 & \\
7 & 3 & $10^{5}$ & 1 & $=$ & & 1 & \\
& & $10^{6}$ & 7 & $=$ & & 7 & \\
\hline
\end{tabular}

Here, the authors of [5] announce the value $n_{3}\left(2,10^{6}\right)=1762$ instead of 1683. In fact, the multiplicities of the superfluous 79 discriminants must necessarily be greater than 2 .

(c) The determination of $n_{p}(p, B)$ is much more difficult, since it involves the complete treatment of various cases of $p$-tuplets of ramified cyclic extensions $N$ of degree $p$ over quadratic fields $k$ of $p$-rank $\rho \geq 1$ and discriminant $\left|d_{k}\right|<B / f^{2}$ with conductor $f>1$, according to Corollary 3.2. If $p=3$, then further contributions arise from Corollary 3.3. Here, $\delta \geq 1$ is possible, and thus the principal $p$ th powers $\alpha_{1}, \ldots, \alpha_{\rho}$ of ideals of $k$ must be examined for each of the relevant quadratic discriminants $d_{k}$.

(d) By Corollary 3.1, $n_{p}(p+1, B)$ is the number of quadratic fields $k$ with $p$-rank $\rho=2$ and discriminant $\left|d_{k}\right|<B$, i.e., $(p+1)$-tuplets of unramified cyclic extensions $N \mid k$ of degree $p$ with conductor $f=1$.

In the case of $p=3$, where exceptionally not only $\left(p^{2}-1\right) /(p-1)=p+1$ but also $(p-1)^{2}=p+1$, we must additionally consider the number of ramified cyclic extensions $N$ of degree $p$ over quadratic fields $k$ of $p$-rank $\rho=0$ and discriminant $\left|d_{k}\right|<B / f^{2}$ with three prime divisors $q_{1}, q_{2}, q_{3}$ of the conductor $f$, such that $w \leq 1$.

A further additive component for $p=3$ is the number of pure cubic discriminants of multiplicity 4 , which can again be evaluated with the aid of Theorem 2.1 by counting products of up to five primes below certain bounds, taking into 
account the parameter values

\begin{tabular}{cc|c|cccccc}
\multicolumn{10}{c}{$(e, t)=(2,2)$ and } \\
\multicolumn{10}{c}{$(e, u, v)=(1,2,1),(1,2,2),(0,3,0),(0,2,2),(0,2,3)}$. \\
\hline$p \quad(p-1) / 2$ & $B$ & $n_{p}(p+1, B)$ & $f=1$ & $d_{k}<-3$ & $f>1$ & $d_{k}=-3$ \\
\hline 3 & 1 & $10^{4}$ & 7 & $=$ & 7 & 0 & + & 0 \\
& & $10^{5}$ & 216 & $=$ & $214+1$ & 0 & + & 2 \\
& & $10^{6}$ & 3216 & $=$ & $3190+$ & 15 & + & 11 \\
5 & 2 & $10^{5}$ & 39 & $=$ & 39 & & & \\
& & $10^{6}$ & 398 & $=$ & 398 & & & \\
7 & 3 & $10^{5}$ & 1 & $=$ & 1 & & & \\
\hline
\end{tabular}

The result $n_{3}\left(4,10^{6}\right)=3216$, in comparison to the number 3189 given in [5], reveals the lack of 27 complex cubic discriminants of multiplicity 4 in the original version of [5].

(e) Finally it should be mentioned, that the authors of [5] announced the extremely exciting number $n_{3}\left(5,10^{6}\right)=7$, which caused a lot of confusion. However, our Example 5 after Theorem 2.1 shows that the minimal occurrence $d_{L}=-16008300$ of multiplicity 5 (which is only possible for pure and certain totally real cubic fields) lies considerably outside the range of the computations in [5].

\section{BIBLIOGRAPHY}

1. I. O. Angell, A table of complex cubic fields, Bull. London Math. Soc. 5 (1973), 37-38.

2. T. Callahan, Dihedral field extensions of order $2 p$ whose class numbers are multiples of $p$, Canad. J. Math. 28 (1976), 429-439.

3. H. Davenport and H. Heilbronn, On the density of discriminants of cubic fields. II, Proc. Roy. Soc. London Ser. A 322 (1971), 405-420.

4. R. Dedekind, Über die Anzahl der Idealklassen in reinen kubischen Zahlkörpern, J. Reine Angew. Math. 121 (1900), 40-123.

5. G. W. Fung and H. C. Williams, On the computation of a table of complex cubic fields with discriminant $D>-10^{6}$, Math. Comp. 55 (1990), 313-325.

6. F. Gerth III, Cubic fields whose class numbers are not divisible by 3, Illinois J. Math. 20 (1976), 486-493.

7. M. N. Gras, Méthodes et algorithmes pour le calcul numérique du nombre de classes et des unités des extensions cubiques cycliques de $\mathbb{Q}$, J. Reine Angew. Math. 277 (1975), 89-116.

8. H. Hasse, Arithmetische Theorie der kubischen Zahlkörper auf klassenkörpertheoretischer Grundlage, Math. Z. 31 (1930), 565-582.

9. __ Arithmetische Bestimmung von Grundeinheit und Klassenzahl in zyklischen kubischen und biquadratischen Zahlkörpern, Abh. Deutsche Akad. Wiss. Berlin 2 (1950), 3-95.

10. P. Llorente and J. Quer, On totally real cubic fields with discriminant $D<10^{7}$, Math. Comp. 50 (1988), 581-594.

11. D. C. Mayer, Table of pure cubic number fields with normalized radicands between 0 and 100000 , Univ. Graz, 1989.

12. __ Table of simply real cubic number fields with discriminants between -30000 and 0, Univ. Graz, 1989. 
13. D. Shanks, Review of I. O. Angell, Table of complex cubic fields, Math. Comp. 29 (1975), 661-665.

14. O. Taussky, A remark concerning Hilbert's Theorem 94, J. Reine Angew. Math. 239/240 (1970), 435-438.

15. B. M. Urazbaev, The distribution of the discriminants of cyclic fields of prime degree, Izv. Akad. Nauk Kazakh. SSR, Ser. Fiz.-Mat., 1 (1969), 8-14. (Russian)

Department of Computer Science, University of Manitoba, Winnipeg, Manitoba, CANADA R3T 2N2

E-mail address: mayer@gold.cs.umanitoba.ca 


\title{
Supplement to
}

\section{MULTIPLICITIES OF DIHEDRAL DISCRIMINANTS}

\author{
DANIEL C. MAYER
}

\section{NUMERICAL EXAMPLES}

Generally, $p=3$ in all examples. The information about the 14 totally real cubic discriminants with multiplicities 4,6 has been taken from [10]. Data for the 69 complex cubic discriminants with multiplicities 6,9 has kindly been made available by the authors of [5] in personal communication.

A common feature of all examples is that the 3-rank of the ring class group $\bmod f$ of the quadratic field $k$ is always $\rho+t+w-\delta(f)=3$, whence the sum of all partial multiplicities equals $\frac{1}{2}\left(3^{p+t+w-\delta}-1\right)=13$.

Both parts $(w \leq 1$ and $w=2)$ begin with the unconstrained cases and are arranged according to increasing quadratic 3-class rank $\rho$ of $k$. Correspondingly, the values of $t+w$ (resp. $t$ ) and of $u$ decrease.

For any integer $n \geq 1$, denote by $\mathcal{O}_{n}$ the suborder $\mathbf{Z} \oplus \mathbf{Z} n \frac{1}{2}\left(d_{k}+\omega\right)$ of the maximal order $\mathcal{O}_{k}$, where $\omega=\sqrt{d_{k}}$. Then the unit group $U\left(\mathcal{O}_{n}\right)$ is exactly the intersection $U_{k} \cap \mathbf{Q}^{\times}(n) \cdot k_{n}^{\times}$.

In the totally real case, the fundamental unit of $k$ is denoted by $\eta$. If $\rho=0$, then the condition $\eta \in \mathcal{O}_{n}$ is certainly sufficient for $\delta(n)=0$.

Part 1. Applications of Corollary 3.2 to conductors with $w \leq 1$.

a) A totally real cubic field with

$$
\begin{gathered}
\rho=0, t=2, w=1, \delta_{\max }=0, u=3=t+w, \\
f=q_{1} \cdot q_{2} \cdot q_{3} \text { with } q_{1}=2, q_{2}=7, q_{3}=3 .
\end{gathered}
$$

Here, we have $I_{k, 3}(f)=U_{k} \cdot k^{\times}(f)^{3}$. There are no constraints from the fundamental unit $\eta$ of $k$, since $\delta\left(q_{1}\right)=0, \delta\left(q_{2}\right)=0$ and $\delta\left(q_{3}\right)=0$. The quadratic discriminant $d_{k}$ is simultaneously congruent $-3(\bmod 9)$ and congruent $5(\bmod 8)$, since $2 \mid f$, $\left(\frac{d_{k}}{2}\right) \equiv 2 \equiv-1(\bmod 3)$

\begin{tabular}{c|cccc} 
No. & $d_{L}$ & $d_{k}$ & $\eta$ & \\
\hline 1 & 8250228 & 4677 & $2796250463+40887672 \omega$ & $\in \mathcal{O}_{2} \cap \mathcal{O}_{7} \cap \mathcal{O}_{3}$
\end{tabular}

The partial multiplicities $m_{3}\left(d_{k}, f^{\prime}\right)$ of divisors $f^{\prime}$ of the conductor $f$ can be determined by means of Corollary 3.2 .

$$
\begin{array}{c|cccccccc}
f^{\prime} & 1 & q_{1} & q_{2} & q_{3} & q_{1} q_{2} & q_{1} q_{3} & q_{2} q_{3} & q_{1} q_{2} q_{3} \\
\hline m_{3}\left(d_{k}, f^{\prime}\right) & 0 & 1 & 1 & 1 & 2 & 2 & 2 & 4
\end{array}
$$

b) 14 complex cubic fields with

$$
\rho=1, t+w=2, \delta_{\max }=0, u=2=t+w,
$$

$$
f=q_{1} \cdot q_{2} \text { with } q_{1}=2 \text {. }
$$

In this case, $I_{k, 3}(f) / k^{\times}(f)^{3}=\langle\alpha\rangle$. There do not arise restrictions from the single generating principal ideal cube $\alpha$ of $k$, i. e., $\alpha \in \mathbb{Q}^{\times}(f) \cdot k_{f}^{\times} \cdot k^{\times}(f)^{3}$, since $\delta\left(q_{1}\right)=0$ and $\delta\left(q_{2}\right)=0$. All quadratic discriminants $d_{k}$ are congruent $5(\bmod 8)$, since $2 \mid f$. The class numbers of all cubic fields $L$ are divisible by 9 .

Typeset by $\mathcal{A}_{\mathcal{M}} S-\mathrm{T}_{\mathrm{E}} \mathrm{X}$ 

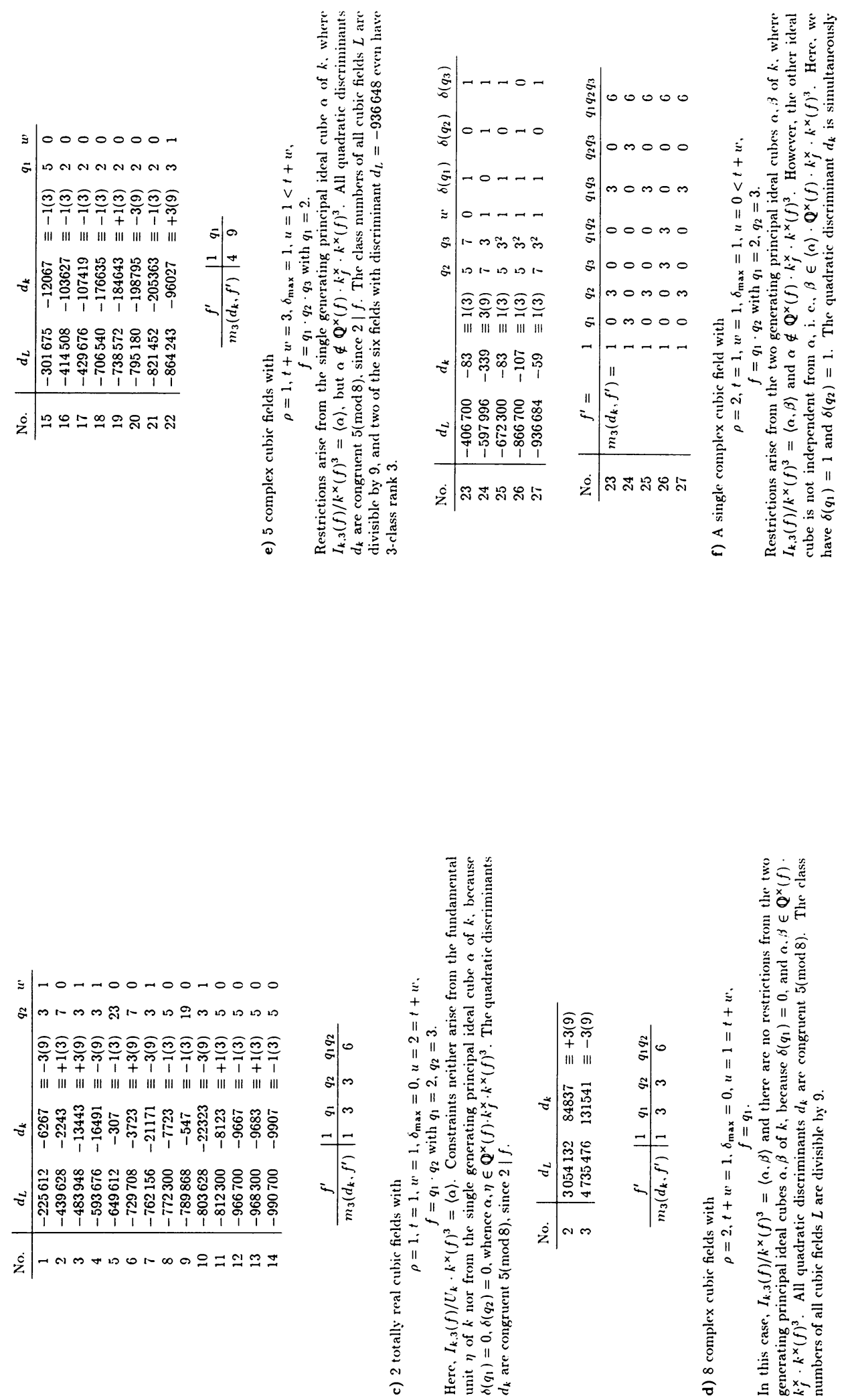


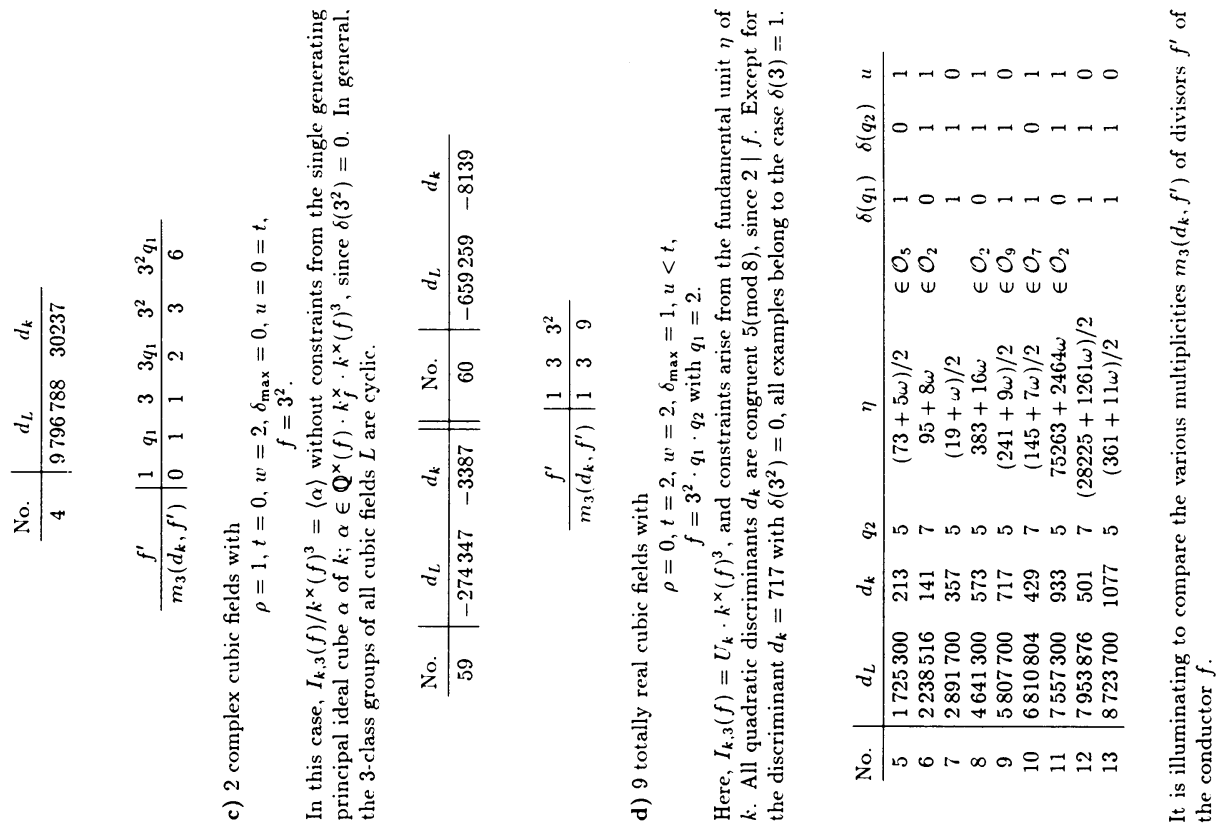



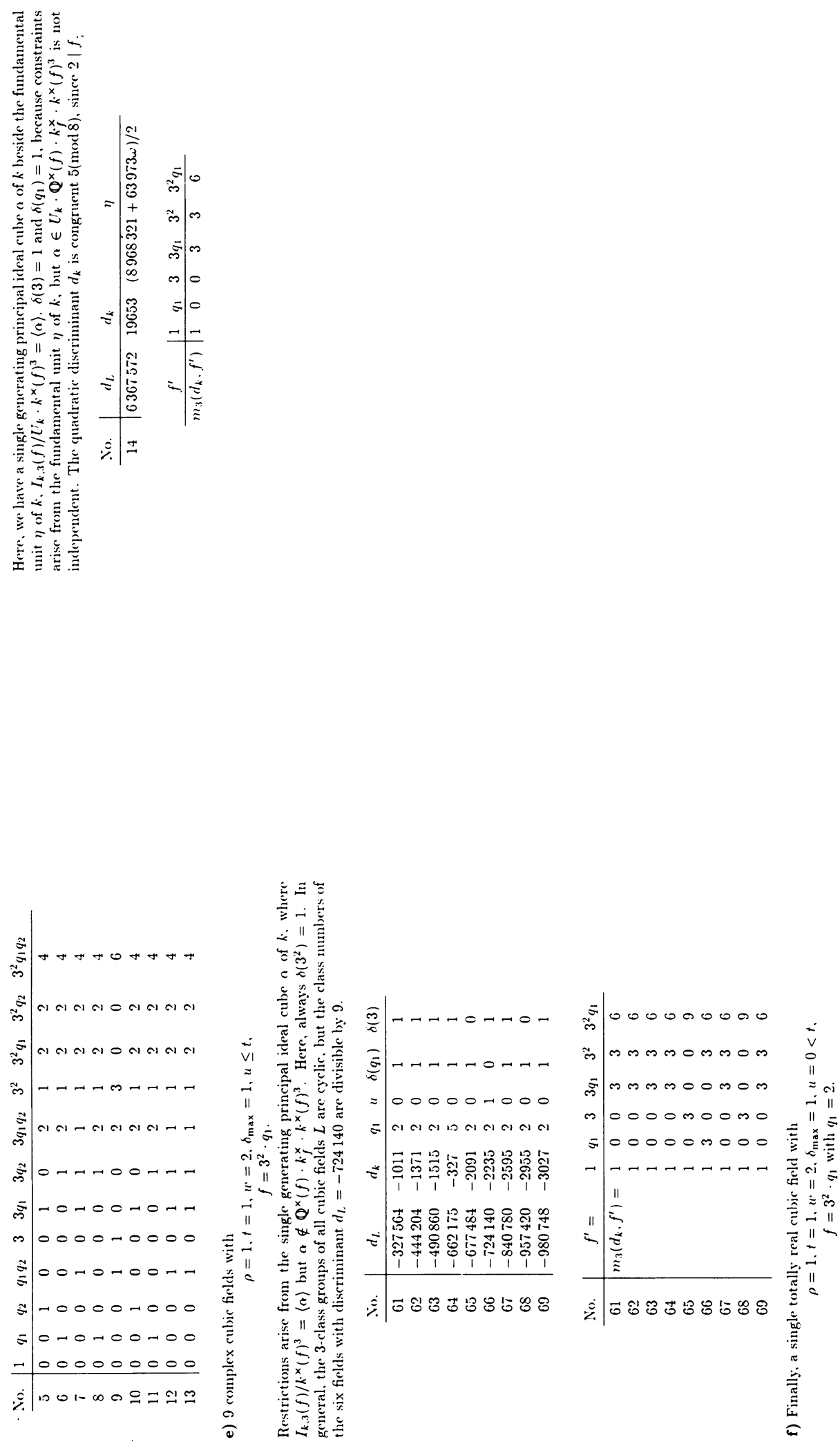